UCRL-JC-106882

PREPRINT

\title{
ELECTROPRODUCTION AT LARGE MOMENTUM TRANSFERS
}

\author{
Karl van Bibber \\ Lawrence Livermore National Laboratory \\ Livermore, CA 94550 U.S.A.
}

\section{This paper was prepared for Conference \\ Procezdings for the "Workshop on \\ Hadronic Physies Beyond 10 GeV" \\ Dourdan, France \\ October 8-12, 1990}

March 1991

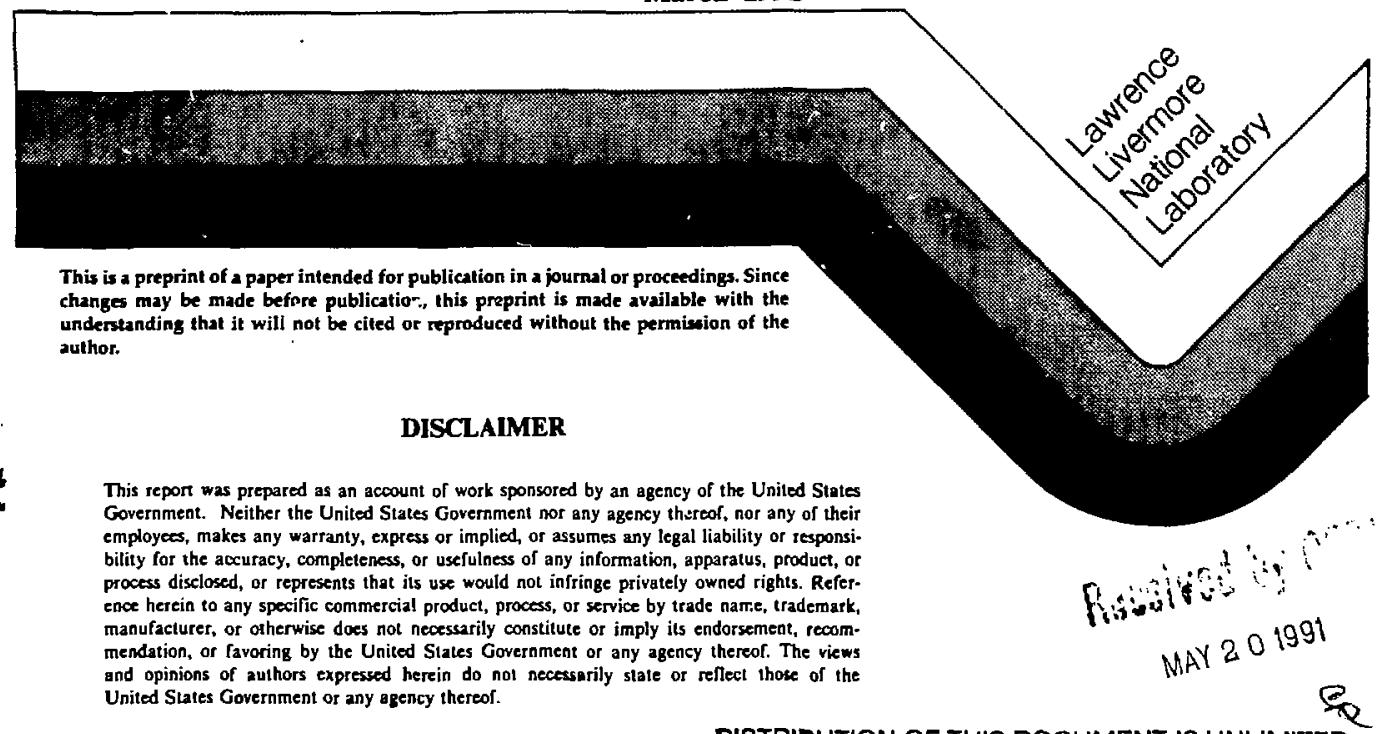

This report was prepared as an account of work sponsored by an agency of the United Stotes Government. Neither the United States Government nor any agency thereor, nor any of their employees, makes any warfanty, express or implied, or assumes any legal liability or responsibility for the accuracy, completeress, or usefulness of any information, apparatus, product, or process diselosed, of represents that its use would not infringe privately owned rights. Reference herein to any specific commercial product, process, or service by trade name, trademark, manufacturer, or ofherwise does not neoessarily constitute or imply its endorsement, recommendation, of favoring by the Uniced States Government of any agency thereof. The views and opinions of authors expressed herein do not necessarily slate or reflect those of the 


\section{DISCI.AJMER}

This document was prepared as an acromin of work sponsored by an apency of the United States Goverameat. Neithes the United Sintes Gorernmeat ker the Unirersity of Calibornin mor any of their employees, makes any warranty, express or implied, or

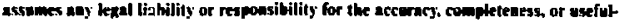
asss of any information, apperatus, preduct, or precess disclesed, of represents thn: its wat would ox infriane privately owned rights. Reference hetein to any specific casonetciel products, process, or service by trade mame, trudemark, manufacturer, or of herwise, does art necessarily constifute or inply its endorsement, recenmendation. or faverine by the United States Government or the University of Callionin. The views and opinions of authors expressed herein do aot mecrsarily state or rellect

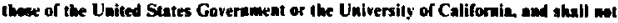
he used for advertisian or product endorsenent purposes.

\section{RTAM!}




\title{
Electroproduction at large momentum transfers
}

UCRL-JC- -106882

Karl van Bibber

DE91 012032

Lawrence Livermore National Laboratory, P.O. Box 808, Livermore, CA 94550 , U.S.A.

\begin{abstract}
The possibilities of electroproduction experiments at a facility such as the proposed European electron accelerator are discussed. Examples given are from studies of hadronization, color transparency, backward production, virtual Compton scattering and target spectator decay. Some conclusions about machine parameters are drawn.
\end{abstract}

\section{INTRODUCTION}

The aim of this report is to outline the opportunities for coincidence electroproduction at an electron accelerator of nominal energy $10-20 \mathrm{GeV}$, as contemplated at this workshop. The point of view will necessarily be an experimental one, with an eye to important machine parameters and detector capabilities.

This section contains brief introductory remarks concerning kinematics, and the scope of this report. Section 2 is a selection of examples of the physics that could be carried out at the future European machine. These are necessarily just a few of many very timely topics in QCD and the structure and dynamics of the nucleon and nuclei. However, due to the unavailability of the combination of energy, beam quality and sophisticated detectors for the foreseeable future, the timeliness of most of these experiments will likely endure the decade it will take before such an accelerator would be operational. In Section 3, the author expresses his preliminary opinion on the desirable characteristics of the facility.

Figure $1 \mathrm{a}$ is a plot of the kinematical range in $\nu$ and $Q^{2}$ accessible to such a machine for a nucleon target, assuming a bearn energy of $E=15 \mathrm{GeV}$, and $E^{\prime}>2 \mathrm{GeV}$ (for reasons of $e / \pi$ separation). The shaded area indicate regions where either $Q^{2}$ is low, or the scattering is to the discrete low-lying resonances ( $W<2 \mathrm{GeV}$ ). The open area is the region where one is cleanly in the Bjorken scaling regime (i.e. point-like coupling to quarks in deep inelastic scattering). The allowed kinematical region for CEBAF (with $E$ $=4 \mathrm{GeV}$ and $E^{\prime}>1 \mathrm{GeV}$ ) is virtually disjoint with the criteria for scaling. In addition to being in the scaling region, we may wish for the energy transfer to the quark $\nu$ to be large, as is the case for the study of hadronization.

To put things in perspective, Figure $I b$ shows the $\nu$ and $Q^{2}$ plane for a high energy muon scattering experiment (EMC, $280 \mathrm{GeV}$ ), with the nominal kinematical region of the European machine shown as the tiny hatched region. The cutoff of the data in the plot is due to a minimum angle cut on the scattered muon.

As the scattered lepton defines the momentum transfer to the target, $q^{\mu}=(\nu, p)$, the outgoing hadron in electroproduction may then be characterized by the fraction of the struck quark's momentum which it carries: $x_{F}=p^{*} / p^{*}$ max , or $z=E_{h} / \nu$, which are numerically almost the same for $x_{F}>0.2$. In this report, a few examples are given deliberately to sample different regions of $x_{F}$, from the exclusive processes $(x=1)$, to the target spectator region $(x=-1)$. Charm electroproduction will be excluded, as it will be dealt with extensively in the talks by Brodsky, Martin and Breton at this workshop. 

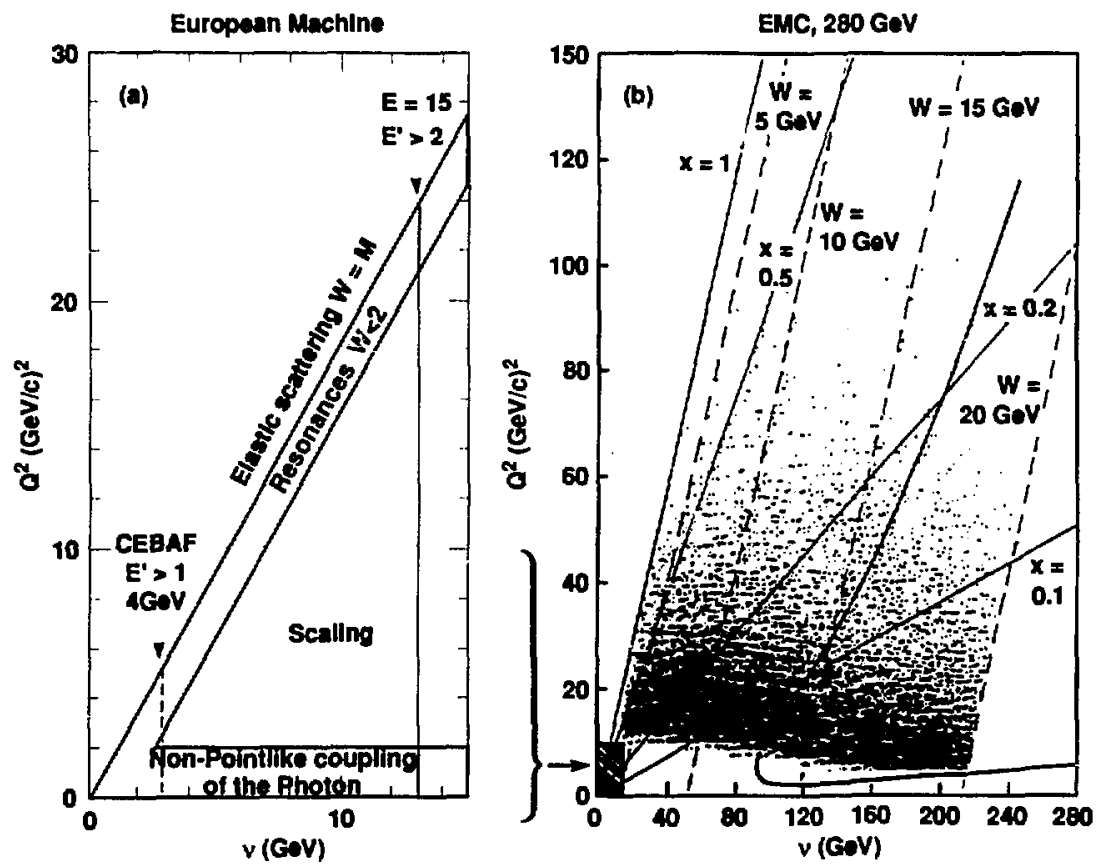

Figure 1. (a) Kinematical range in $\nu$ and $Q^{2}$ accessible to the proposed new European electron accelerator. The unshaded region corresponds to Bjorken scalinf. (b) Kinematical range for the EMC experiment.

\section{SPECIFIC EXPERIMENTS}

\subsection{Studies of Hadronization}

Figure 2 shows the familiar plot of the attenuation ratio of fast hadrons for $z>0.2$. The ratio here is made between copper and deuterium targets, and is a function of $\nu=E-E^{\prime}$. The lowest- $\nu$ point is from SLAC [1] the remainder from EMC [2]. The studies of the $A$-dependence of hadronization ought to reveal the space-time development of hadrons in deep inelastic scattering, insofar as differing thicknesses of nuclear matter are interposed in which a struck quark or nascent hadron could be absorbed.

Figure 2 shows us that for $\mathrm{Cu}$ at least, the ratio falls dramatically below $30 \mathrm{GeV}$. Two questions are raised: how is attenuation information connected to the physical description of hadronization, and what demands are placed on statistical and systematic errors of the experiment? 


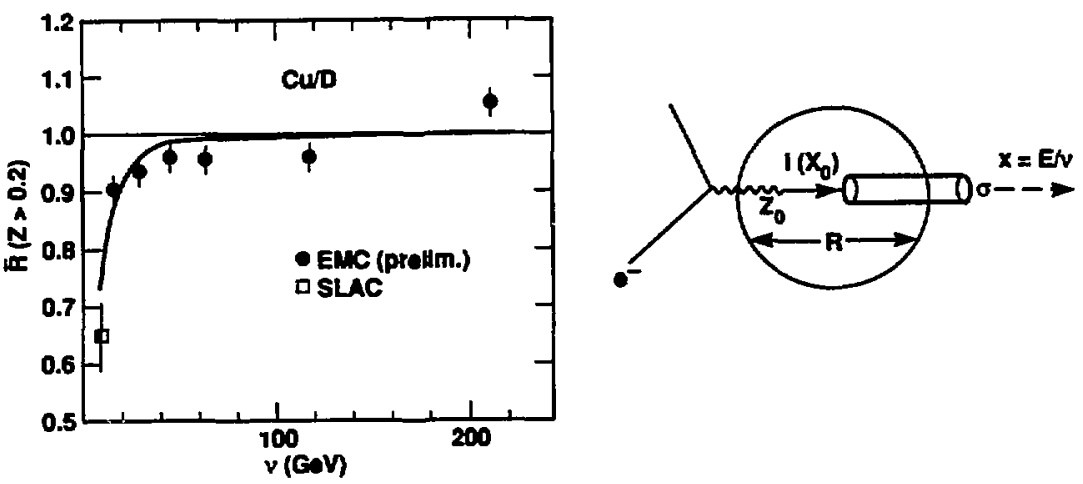

Figure 2. Ratio of cross sections per nucleon for the production of all charged hadrons, between copper and deuterium targets. See text for details.

In way of exploring the second question, let us assume we are privy to knowledge of the basic picture of hadronization (that sets a bound as to how easy it could ever be). For purposes of demonstration the picture is one where the struck quark recoils and the string between the quark and diquark fragments according to e.g. the Lund string model. The production of the leading hadron may be suppressed by one of two mechanisms: (a.) the current quark may inelastically scatter, prior to hadronization, or (b.) the hadron produced may inelastically scatter.

Regarding the interactions of the current quark, analysis of the EMC data show that the effective quark-nucleon cross section $\sigma_{q N}$ is bounded by $5 \mathrm{mb}$, and is consistent with 0 (Figure 3a). (The family of curves corresponding to each value of $\sigma_{q N}$ represent different assumptions about the formation length, and are clearly irrelevant to this conclusion.) One does not know what will happen belo'v $20 \mathrm{GeV}$; one should be prepared that there may be a critical energy below which it will rise steeply.

With respect to the formation length $c r$, it is an invariant feature of all models that it should be linear in $\nu$ (it enters into the Lorentz factor in any model). On general grounds, the QCD string tension $\kappa$ being $\approx 1 \mathrm{fm} / \mathrm{GeV}$, one expects that the formation length will become comparable to nuclear dimensions below $20 \mathrm{GeV}$ or so, and the resulting attenuation to be rapidly varying in that region. Beyond that, it may depeno on the hadron mass and/or $z$. Even in the Lund string model, $c \tau=g(z) \nu$, there is ambiguity about when the hadron exists [3]. One may posit the hadron to exist at the time of the formation of the first $q \bar{q}$ string break from which it results (called the constituent length $l_{c}$ ), or when both constituents exist and oscillate past one another for the first time (called the yo-yo length, $l_{y}$ ). The difference between these formation lengths increase with $z$ and is maximal at 1 , where the statistics are the worst (see Figure $3 \mathrm{~b}$ ). 

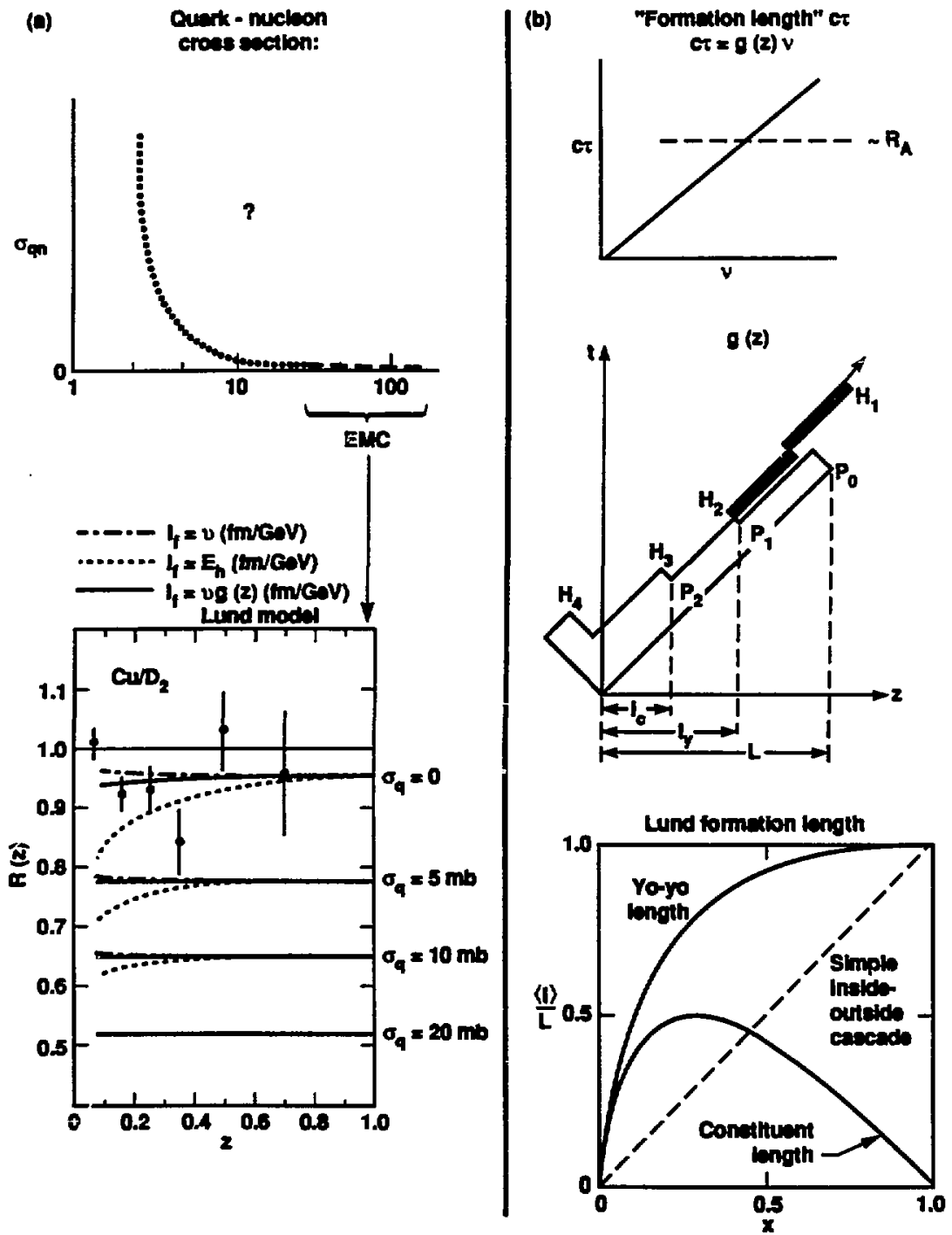

Figure 3. (a) Attenuation ratio for $\mathrm{Cu} / \mathrm{D}_{2}$ (data from $E M C$ ) vs. $z$, and families of calculations ordered by $\sigma_{\mathrm{gNN}}$. (b) Formation length within the Lund string; note the ambiguity in its dependence on $z$ [3]. 
How would one independently deterrinine the formation length $c r$ and the quarknucleon cross section $\sigma_{q N}$ in an energy regime where they both may be sharply energy dependent? The answer is that while the measurement of the attenuation in a single nuclear target yields a continuous degeneracy of values of $\mathrm{cr}$ and $\sigma_{q N}$, the family of such curves resulting from the measurement of several target anclei must intersect at one point (Figure 4a). Realistic relative errors produce a diamond-shaped region of constraint (Figure $4 \mathrm{~b}$ shows the effect of $1 \%$ errors). One wishes to perform this separation then, in suitably small regions $\Delta y \Delta z(\approx(1 \mathrm{GeV}) \times(0.1))$, as well testing for $p_{\perp}$ and $m_{h}$ dependence. The requirements of such an experiment are estimated to be an integrated luminosity of at least $10^{39}$ and tight control of systematic errors in performing the $A$ dependence. It is intriguing that the MIT-SLAC data [1] seem inconsistent with any $\sigma_{q N} \geq 0$, even allowing for their large errors (Figure 4c). N. Pavel of EMC [4] points out the inconsistency of the combined EMC and SLAC data set even with an extended variation of this model. Gyulassy and Plümer [5] in arriving to the same conclusion, propose a 'string-flip' model which challenges the notion of the independence of the fundamental hadronization process within the nuclear medium; similar conclusions are found by Kopeliovich [6]. In any case, it seems that any proper description of hadsonization with nuclear targets must take account of the coherence of the quark's interaction over all possible partons in its path [7].

\subsection{Color Transparency}

Suggestive evidence for the phenomenon of color transparency has been found in quasielastic pp scattering at BNL [8]. An experiment to search for it in quasielastic ep scattering is scheduled to run at SLAC, which will be free from possible complica. tions peculiar to hadron scattering [9]. While color transparency would seem to be a corollary of the same QCD physics which gives rise to the very successful dimensional counting rules, one must remember that an unambiguous demonstration is yet to come. Should the prediction be borne out in quasielastic scattering, the phenomena should be sought for in as many channels as possible, particularly exclusive meson electroproduc. tion $\left(e, e^{t} h\right), h=\pi, K, \rho^{0} \ldots$ etc. and its kinematic dependence on $Q^{2}, t$ determined. In this way, one stands to learn details about the QCD expansion of the small hadron, and which channels do in fact proceed through production of an initially small color singlet.

Ralston and Pire have extended the concept of color transparency to the hypothesis of nuclear filtering [10]. The statement of this hypothesis is that hard QCD processes will always appear purer in a nuclear target than in a nucleon target, as soft $Q C D$ amplitudes will have been filtered out. This is effectively synonymous with the statement that hard QCD proceeds through small hadrone, and soft QCD through normal-size hadrons. As an example, we show the potential sensitivity of the measurement of a self-analyzing decay in examining this idea. Exclusive rho electroproduction $p\left(e, e^{\prime} \rho^{0}\right)$ has been studied extensively at low- $Q^{2}$ at Cornell, DESY, SLAC and more recently at high- $Q^{2}$ by EMC. These experiments measure the transverse or longitudinal nature of the rho, as revealed by its decay angular distribution $\rho^{0} \rightarrow \pi^{+} \pi^{-}$. The picture which emerges is that only 

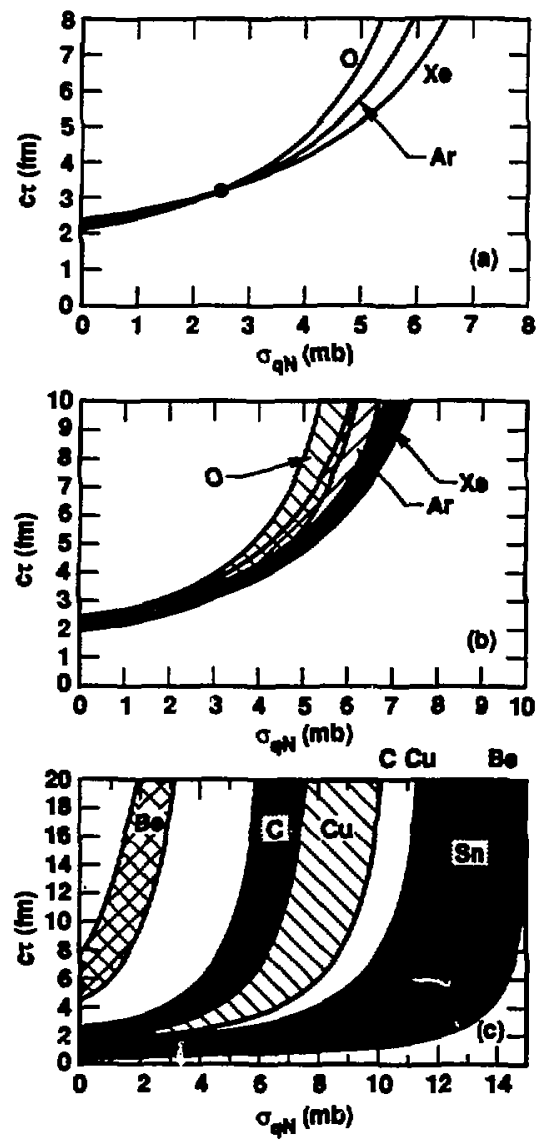

Fig:rre 4. (a) Separation of the formation length $c T$ and quark-nucleon inelastic cross section $\sigma_{q N}$ via the $A$-dependence of nuclear attenuation. (b) As in (a), but with $1 \%$ relative errors between different targets. (c) Plot for the MIT-SLAC data [1].

transversely polarized $\rho^{0}$ 's are produced at the real photon point, with a continuous evolution to purely longitudinally polarized $\rho^{0}{ }_{\mathrm{s}}$ somewhere above $Q^{2}=10(\mathrm{GeV} / \mathrm{c})^{2}$ (and the value of $50 \%$ is achieved around $Q^{2}=2$ ). For purposes of the exercise, let us suppose that the longitudinally polarized rho's (L) are produced via an initially small color singlet, i.e. will manifest color transparency, whereas the transversely polarized 

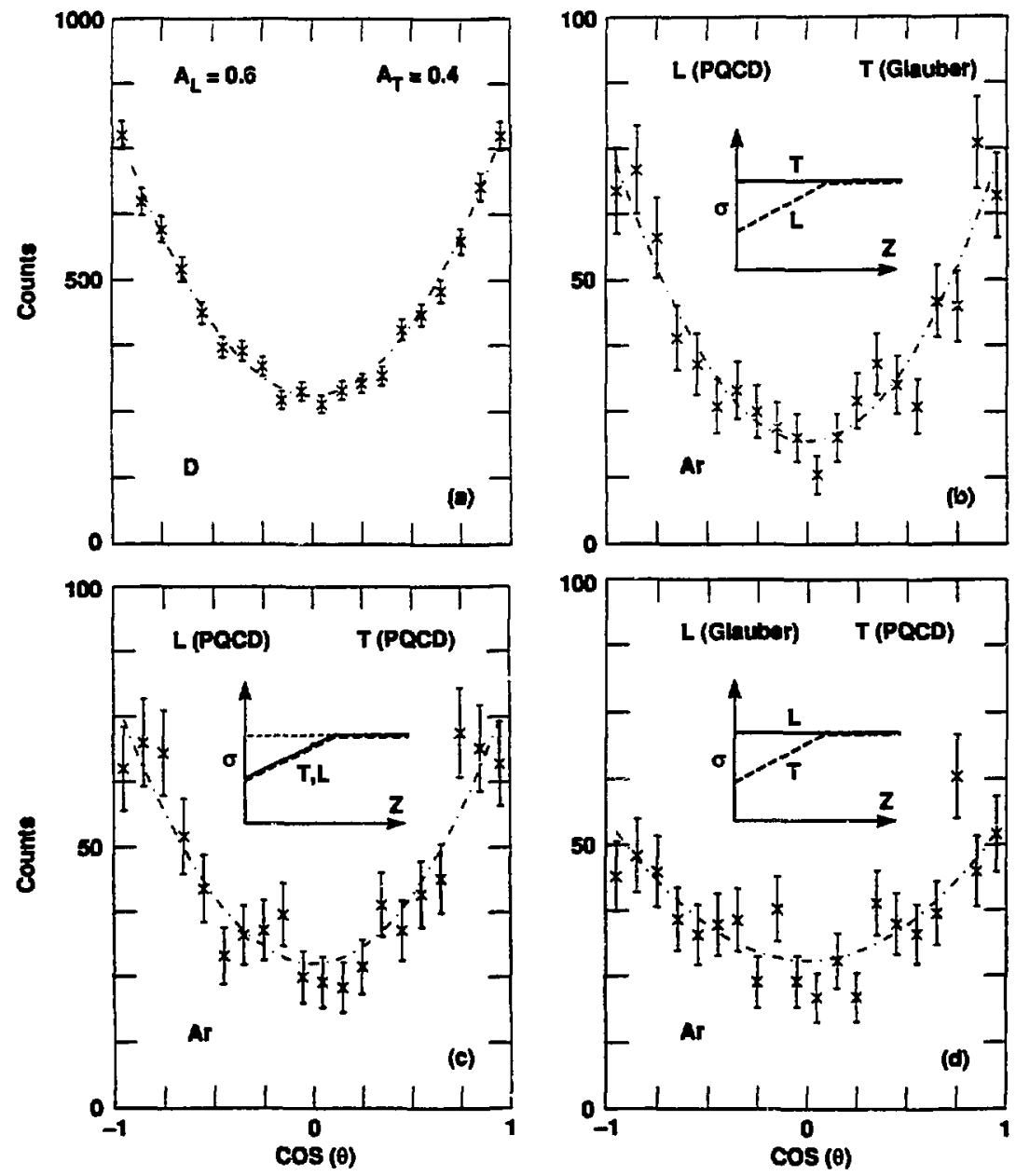

Figure 5. Synthetic data for the decay angular distribution $\rho \rightarrow \pi^{+} \pi^{-}$, and least-squares fit to them. (a) For D. For ${ }^{40}$ Ar: (b) L(pQCD), T(Glauber); (c) L(pQCD), T(pQCD); (d) $\mathrm{L}$ (Glauber), $\mathrm{T}(\mathrm{pQCD})$. Statistics and helicity composition appropriate to the kinematical range $Q^{2}=3-4(\mathrm{GeV} / \mathrm{c})^{2}, W=3-4 \mathrm{GeV}$.

rho's (T) are always produced large, i.e. will undergo the attenuation expected in a simple Glauber model calculation. (This is the behavior expected within pQCD [11]. However, we are not beholden to such theoretical prejudice, and we will consjder all 
combinations!) Figure 5 shows the rho decay angular distributions, in the rho centerof-mass with respect to its recoil direction, for a deuteron target (effectively isoscalar nucleon) and for an Ar target. In this simulation the three angular distributions for the Ar target correspond to different assumptions about the color transparent or Glauber nature of the nascent rho, as labeled by the ideograms. The exact details concerning the initial size and evolution of the small hadrons are from Farrar et al [12], but are not important for this exercise. The statistics result from a luminosity-efficiency product of $5 \times 10^{38}$ nucleous- $\mathrm{cm}^{-2}$ for deuterium, and $7.3 \times 10^{37}$ for argon. Comparing Figures $5 \mathrm{a}$ and $5 \mathrm{~b}$, one sees that the preferential filtering of the $\mathrm{T} \rho^{0}$ relative to the $\mathrm{L}$ leads to a steepening of the anguiar distribution which can be seen by eye. The converse assumption leads to a flattening of the angular distribution from a nuclear target; see T'igure 5d. Figure 6 shows characteristically $3 \sigma$ separation between color transparent and Glauber behavior in individual helicity states, as back-fitted from the synthetic data.

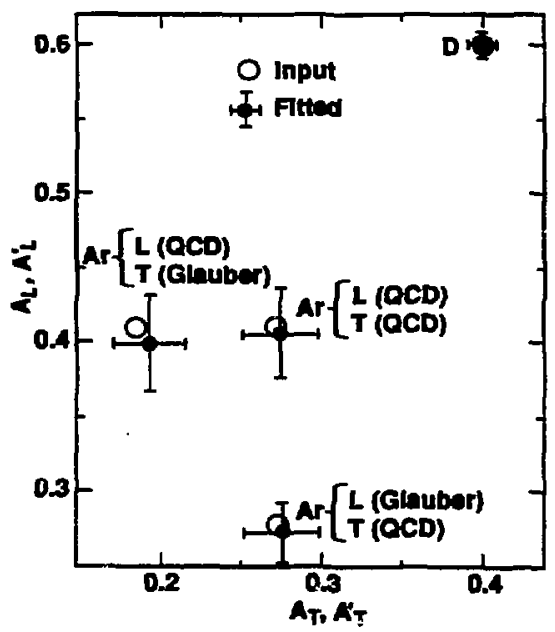

Figure 6. Actual and fitted coefficients of the longitudinal and transverse components to the decay angular distribution $W_{L, T}(\cos \theta)$ from the parent (D) distribution $A_{L, T}$, and the nuclear attenuated distributions $A_{L, T}^{\prime}$.

Other self-analyzing decays may reveal 'nuclear filtering' and thus allow a separation of hard and soft QCD processes. The long-standing issue of the origin of the transverse polarization of the $\Lambda$ observed both in $p p$ and in ep scattering comes to mind. Large polarization effects are expected to be negligible in partonic subprocesses. If true, $\Lambda$ polarization ought largely to vanish when performed in nuclear targets. However, one should be open to surprises. 


\subsection{Tagged Structure Functions}

It has long been recognized that the kinematic domain for nucleons of high momenta $(p \geq 200 \mathrm{MeV} / c)$ emitted in the backward direction $\left(\theta \geq 90^{\circ}\right)$ is of special interest, insofar as it is forbidden for free $N N$ scattering. (The case of single energetic backward nucleons is just one facet of the much broader topic of cumulative production. It is impossible to do justice to cumulative production in its entirety here.) Figure 7 [13] summarizes only part of this data. At sufficiently high bombarding energies, the proton momentum spectra, at $180^{\circ}$ consists of two components, a low momentum component (consistent with the single particle momentum spectrum in nuclei), and a much harder component extending out to at least $1 \mathrm{GeV} / \mathrm{c}$. This component is observed to be independent of the projectile (photons, leptons, hadrons...) and incident energy, ałove some minimum value.
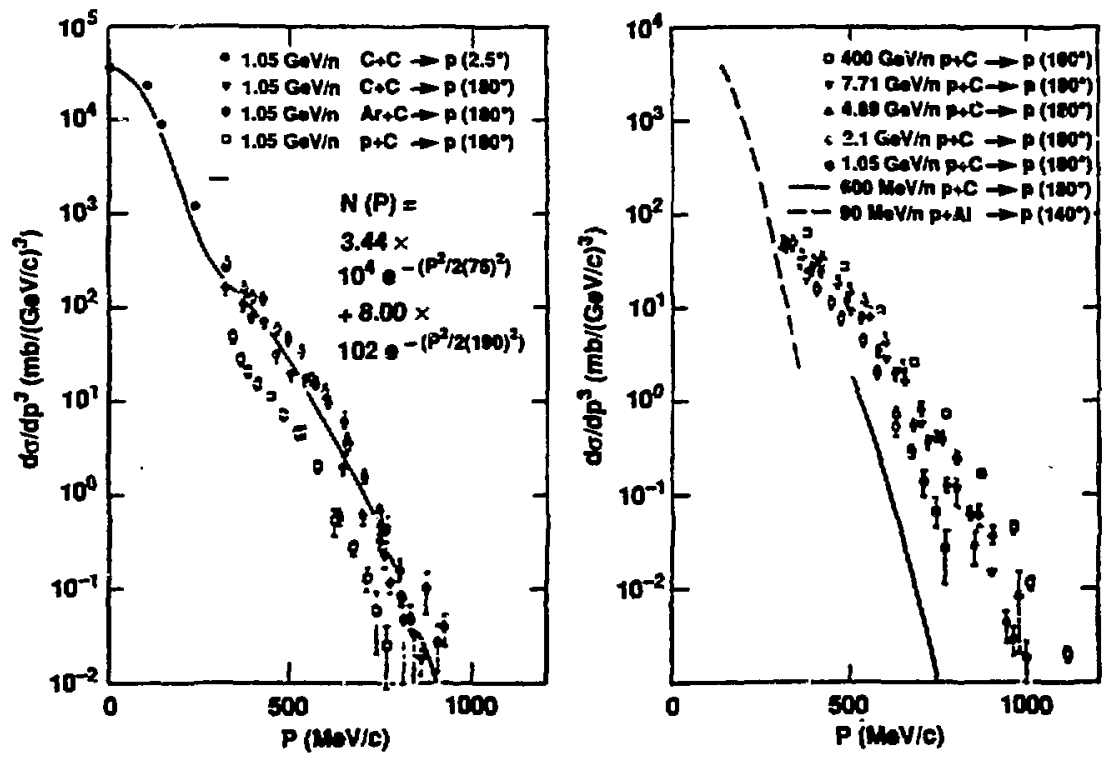

Figure 7. (a) Inclusive proton production at $180^{\circ}$, for various projectiles at 1.05 $\mathrm{GeV} /$ nucleon. (b) Inclusive bacikward proton production with proton beams, various energies. From Geaga [13].

The connection between this scaling component and the short-range $N N$ correlatic 1 was suspected early on, but it was one of several models current at that time. Frankfurt and Strikman [14] proposed a key test for this ides. In their picture, the incident probe 


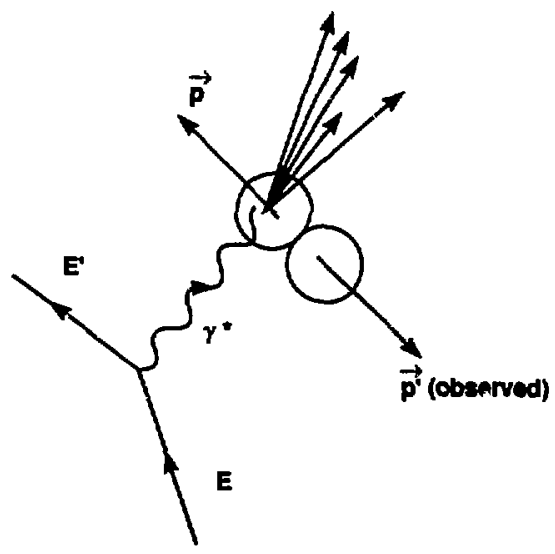

Figure 8. Backtrard production in lepton scattering, according to the modis of Frankfurt and Strikman.

interacts with the partner of the nucleon which is ultimately observed; the backwara nucleon is the spectator in the scattering (Figure 8).

As deep inelastic lepton acattering measures the momentum distribution of the struck parton, the model of Frankfurt and Strikman predicted a softening of the structure function measured in coincidence with high momentum backward nucleons. Furthermore, assuming the correlated $N N$ pair was on the average at rest in the target nucleus, the 'tagged' structure function is connected to the free isoscalar $F_{2}(x)$ by a simple kinematical rescaling:

$$
F_{2}\left(x, p_{3}\right) \propto \frac{1}{2}\left[F^{m}\left(\frac{x}{2-\alpha}\right)+F^{p}\left(\frac{x}{2-\alpha}\right)\right]
$$

Here the light-cone variable $\alpha$ describes the detected nucleon's momentum and in the laboratory frame assumes the value

$$
\alpha=\left[\sqrt{M^{2}+p^{2}}-p_{3}\right] / M .
$$

In the previous expressions, $p$ and $p_{3}$ represent the magnitude of the vector momentum of the backward nucleon, and its component along the direction of the virtual photon (or $W^{ \pm}$in the case of charged current scattering).

The first evidence for such a nuclear-correlation effect was seen in a charged-current scattering experiment in the FNAL 15' bubble chamber filled with a H-Ne mixture [15]. 
As the aata sample was very small, it was not possible to measure the tagged structure function with any meaningful statistics. However, the average value of Bjorken $x$ for events containing a backward proton was $0.17 \pm 0.02$, lower than the value of $0.23 \pm$ 0.01 for the whole sample. Later work by the BEBC collaboration [16] with $D$ as a target agreed well with the Frankfurt-Strikman model, bui again with low statistics. Their data with Ne however, disagreed both with the model and with the FNAL data.

The kind of statistics possible with a high duty factor facility and modern detectors is shown in Figure 9. Shown is the ratio of the tagged structure function with a nitrogen target for $p \geq 200 \mathrm{MeV} / \mathrm{c}$, to the isoscalar nucleon, as a function of $x_{B j}$. The statistics result from the assumption of an integrated luminosity of $2 \times 10^{38}$ nucleon-cm ${ }^{-2}$, and a neutron time-of-flight wall around $180^{\circ}$ with solid angle-efficiency product of $0.025 \mathrm{msr}$. It must be remembered that the dramatic modification shown contains no more physics than the rescaling of the free structure functions. What would be of great interest would be to find deviations from that prediction, indicative of non-trivial modifications to the free structure functions for two nucleons in short-range correlation.

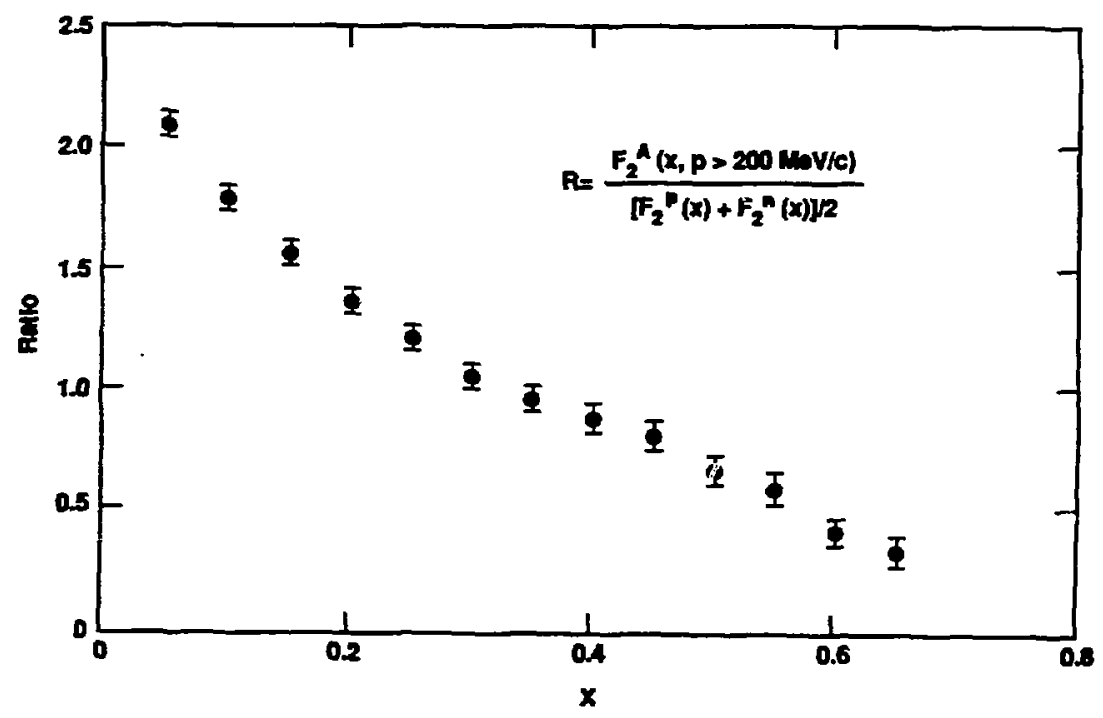

Figure 9. Simulation of the ratio of tagged to free nucleon isoscalar structure functions for $p \geq 200 \mathrm{MeV} / \mathrm{c}$. The target is nitrogen; sce text for details.

One should be forewarned that there may well be strong final state interaction effects in addition to the pair-correlation mechanism even for light nuclei. A more sophisticated treatment of this and related experiments is given by Strikman [17]. 


\subsection{Direct Photon Production}

Measurements of direct photons in electron scattering holds promise to be a powerful probe of the structure of the nucleon. Our best constraints on the quark distribution amplitude of the nucleon come from the simplest and cleanest electromagnetic channels, i.e. form factor measurements $\gamma^{*} p \rightarrow p$, and to a lesser extent, Compton scattering $\gamma p \rightarrow \gamma p$, and photon-photon interactions, $\gamma \gamma \rightarrow p \bar{p}$. The proton elastic form factor measurements suggested the possibility of highly asymmetric quark wavefunctions, e.g., those of Chernyak, Ogloblin and Zhitnitsky (Figure 10). But the situation is far from clear, and the questions presents itself: which probe or probes will be the most discriminating among various quark wavefunctions, if one can indeed validate the use of perturbative QCD methods in deriving them?

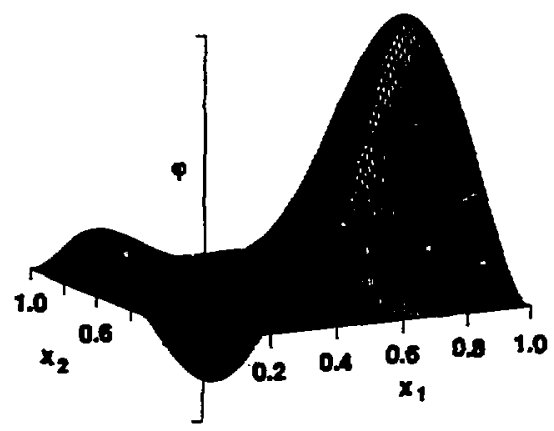

Figure 10. Proton distribution amplitude of Chernyak, Ogloblin and Zhitnitsky [18].

There are reasons to think that virtual Compton scattering experiments, when it is possible to perform them with sufficient statistics and large momentum transfers, may powerfully constrain models of the nucleon. Central to this thinking is the fact that the scattering ep $\rightarrow e^{\prime} p \gamma$ receives contributions not only from the Compton amplitude (Figure 11a), but from the Bethe-Heitler amplitudes (Figure 11b) which are physically indistinguishable, and must be added coherently. As the Bethe-Heitler diagrams may be taken to be purely real, this presents the possibility of the phases of individual Compton amplitudes to become physical observables. (Additionally the photon virtuality $Q^{2}$ is a new degree of freedom, along with $s, t$.) Two issues must then be addreased. First is a practical experimental one, namely, to identify which measurables reveal the phase structure of the Compton proceas. Second is whether it is to be expected that the phasea $\phi=\phi\left(s, t, Q^{2}\right)$ are likely to be very model-dependent and whether the amplitudes and phases will vary markedly with $Q^{2}$. 
Concerning the experimental issue, the charge symmetry properties of the amplitudes play a key role. Note that if one changes the sign of the beam $\left(e^{+} \rightarrow e^{-}\right)$, the Compton amplitude $T_{C}$ changes sign whereas the Bethe-Heitler amplitude $T_{B H}$ does not $\left(T_{C} \rightarrow-T_{C}, T_{B H} \rightarrow+T_{B B}\right)$, as is clear by counting the photon vertices on the lepton. Thus if one has the possibility of both $e^{ \pm}$beams, the asymmetry $\sigma\left(e^{+}\right)-\sigma\left(e^{-}\right)$at any kinematics directly gives the Bethe-Heitler/Compton interference term $2 \operatorname{Re}\left(T_{C} T_{B H}\right) \equiv T_{C / B H}$. (While not the topic of this section, this property pertains to the inclusive virtual Compton scattering in the scaling regime as well, plus an additional property. As in the inclusive (deep inelastic) case, the quark-level Compton radiation must take place from the same parton of charge $q_{i}$ as is struck by the virtual photon, the Compton amplitude is proportional to $e q_{i}{ }^{2}$, whereas the Bethe-Heitler amplitude at the same kinematics goes like $e^{2} q$. From this derives a new charge-cubed (thus valence only) structure function in the interference term, and a new sum rule based on exactly conserved quantum numbers [19]. At least in the inclusive virtual Compton case, the $\sigma\left(e^{+}\right)-\sigma\left(e^{-}\right)$asymmetry is expected to be of the same order of magnitude as each term individually.)

Should positron beams not be available, phase observables may still be contained in the azimuthal dependence of the real photon about the momentum transfer direction $\vec{q}$, keeping $s, Q^{2}, t$ fixed.

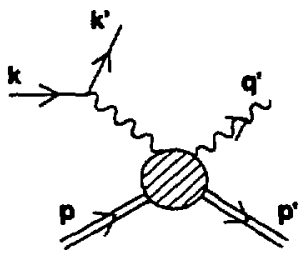

(a)

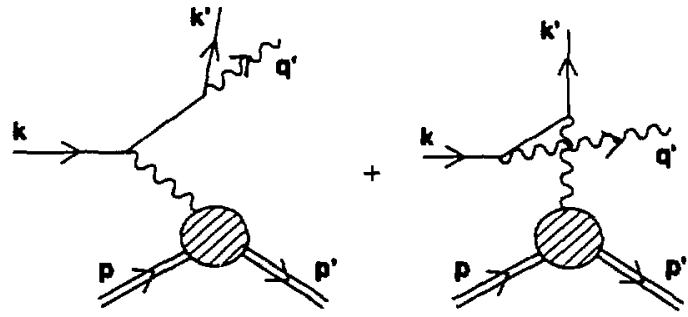

(b)

Figure 11. Diagrams of (a) virtual Compton, and (b) Bethe-Heitler processes for ep $\rightarrow$ ep $\gamma$.

With regard to nucleon models and Compton scattering, the world's real Compton data at large $s, t$ are shown compared with perturbative QCD (pQCD) calculations [20] (Figure 12a) and within a di-quark model [21] (Figure 12b). The PQCD cross section shows a marked difference in magnitude between an asymptotic (symmetric) and nonanymptotic (anymmetric), quark wavefunctions which are represented by the dot-dashed and solid curves respectively in Figure 12a. The non-asymptotic form is larger but still falls a factor of 3-5 short of the data. The di-quark model can reproduce the data more 

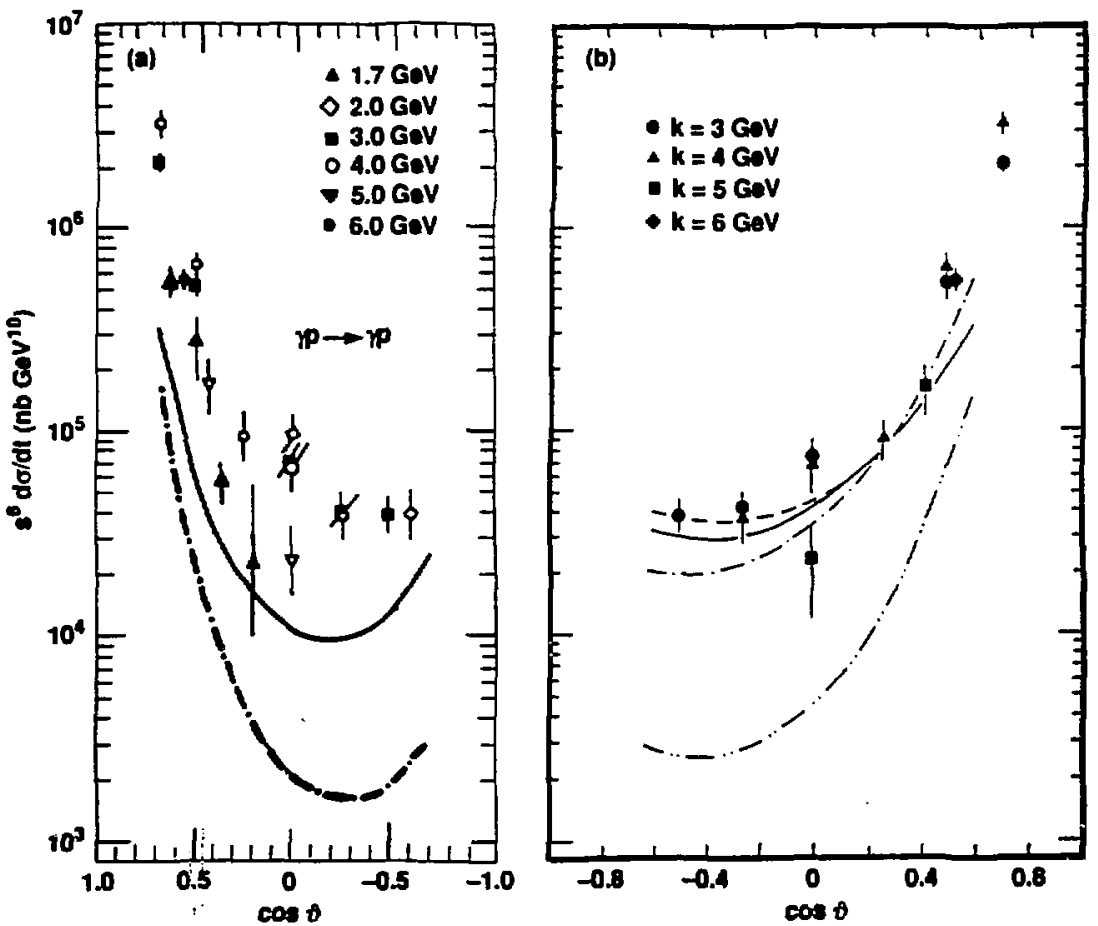

Figure 12. Real Compton data compared with (a) perturbative QCD calculations, (dotdash, asymptotic wavefunction; solid, non-asymptotic) [20], and (b) the di-quark model calculations (solid, dashed, dot-dashed) [21]. The dash-dot-dot curve is a pQCD calculation.

or less. What is more interesting however, is the behavior of the phase for a particular helicity amplitude in the scattering, e.g. $\gamma_{R} p_{+} \rightarrow \gamma_{R} p_{+}$vs. $t$ (or $\cos \theta^{*}$ ) (Figure 13c) for the asymptotic and non-2symptotic forms, which are strikingly different, and in general are different yet for the di-quark model. Farrar and Zhang [22] extended the pQCD calculations to virtual photons, and the results are evidently very rich in behavior (Figure 14). The gQCD first predicts all the physica to scale in the ratios $Q^{2} / \mathrm{s}, t / \mathrm{s}$, and all cross sections to scale as $s^{6} d \sigma / d t$ as expected from dimensional counting rules. But further, the amplitudes change markedly even with the least virtuality explored in these calculations, and sharp behavior in the phese $\phi$ is ween both as a function of $Q^{2} / s$ and $t / s$. (We are not aware of any calculation for $Q^{2}>0$ in a di-quark model yet.)

The point of this is not to endorse any particular theoretical model, or base any 

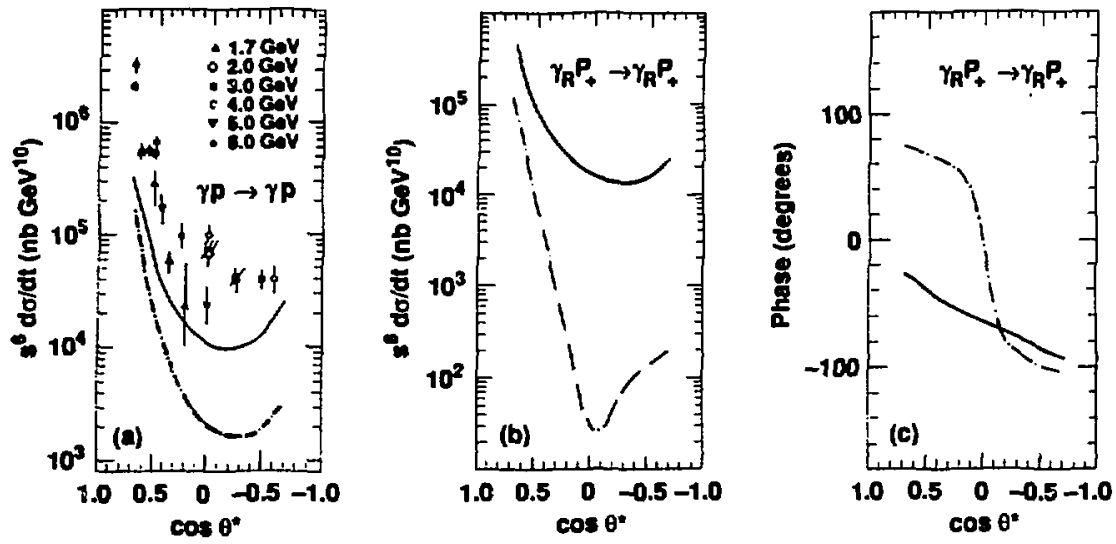

Figure 13. (a) Real unpolarized Compton data with pQCD caiculations for both asymptotic and non-asymptotic quark wavefunctions; (b) cross sections, and (c) phases for the specific helicity channel $\gamma_{R} p_{+} \rightarrow \gamma_{R} p_{+}[20]$. For all three panels the key is as in Figure 1\%.

proposal on the assumptions of e.g. perturbative QCD being a valid description of reality at the kinematics accessible to the machine proposed. Rather it is to motivate the idea that different models may have strikingly different behavior at the amplitude and phase level, which may be testable in high-quality measurements at large momentum transfers. Nature in the end chooses her own amplitudes and phases. The program of "engineering calculations" demonstrating the sensitivity of these interference terms to particular physical models is well underway, some of which are presented at this workshop [23]. Clearly the full program will require polarized beams and targets, and ideally the European machine should have positron capability.

\subsection{Decay of the Target Spectator}

The final example concerns the decay of the target spectator after deep inelastic scattering from a nuclear target. In deep inelastic scattering from a nucleon, one envisions the formation of a tlux tube or gluonic string after the initial lepton-quark scattering. Due to the complexity of non-perturbative QCD, however, it is not obvious (i) what effect the nuclear medium has on the intrinsic hadronization process, or (ii) what the excitation of the nuclear target spectator will be. As mentioned in Section 2.1. there is speculation that the hadronization process may be fundamentally altered by a 'stringflip' mechanism [5], so that the reaulting fragmentation function from an atomic nucleus $D_{h}^{A}(z)$ will in no way be connected to the fragmentation function from a nucleon $D_{h}^{N}(z)$ 

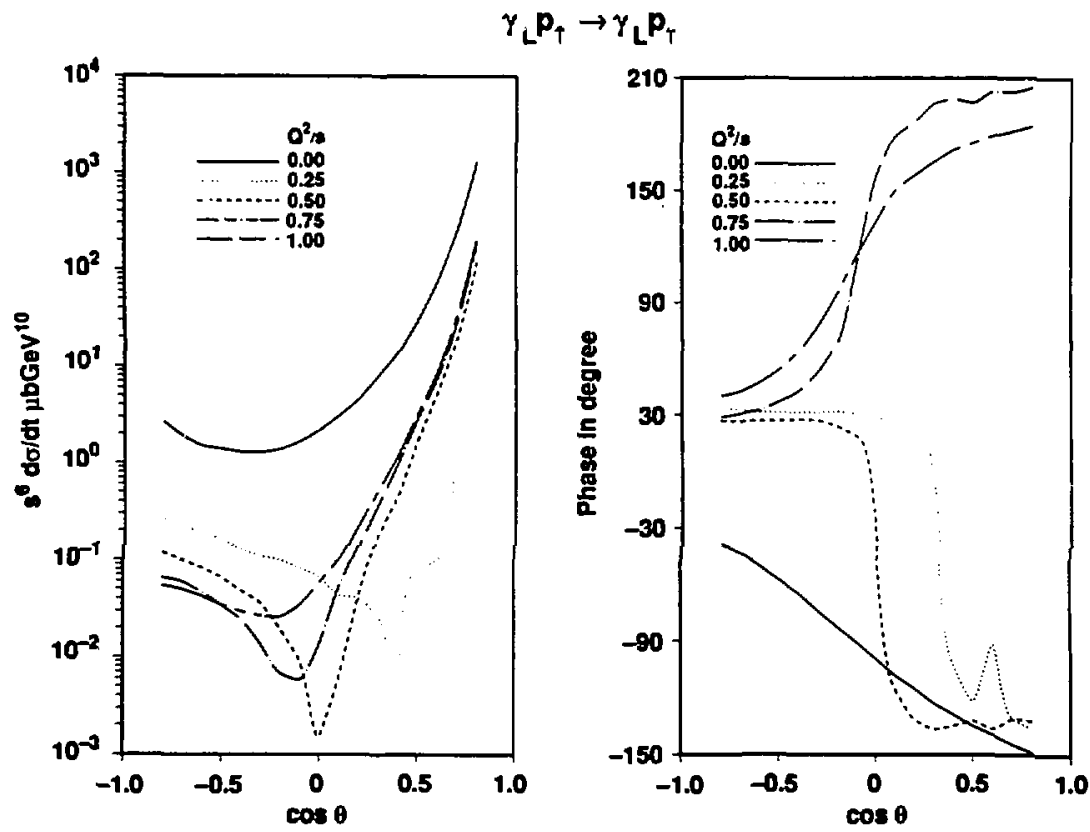

Figure 14. Calculations of virtual Compton scattering in pQCD [22]: (a) partial cross sections, and (b) phases for the helicity amplitude $\gamma L p_{\uparrow} \rightarrow \gamma L P_{\uparrow}$, as a function of $Q^{2} / s$.

which has been modified by the effects of cascading and attenuation. With respect to (ii), there are a few possible mechenisms for excitat:on of the target spectator. The first is the result of an intranuclear cascade due to the interactions of either the current quark or the hadrons that materialize from the string within the nuclear volume. The second results from the flux tube becoming transversely deconfined in a nuclear medium, thereby producing excitation by color polarization as pictured by Zingman and Kerman [24]. Third, the 'string-flip' calculation described above makes a specific prediction for the energy left in the target, based on the fact that the energy of each stringlet (of which there are expected to be several on the average in a medium weight nucleus) is $\kappa R$, where $\kappa$ is the QCD string tension and $R$ is the length of the stringlet.

From an experimental point of view, we simply have no data at present on the state of the nucleus after deep-inelastic scattering. There is not even qualitative information as to whether the quark fragmentation leaves the nucleus virtually unexcited, or highly excited. A firat attempt to gain come insight on target excitation has gotten underway, however. A new component has been added to the muon scattering experiment E-665 
(a)

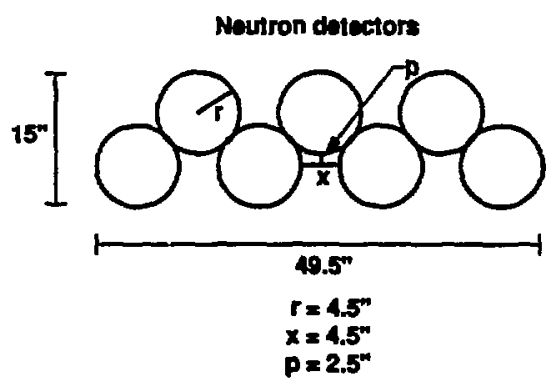

(b)

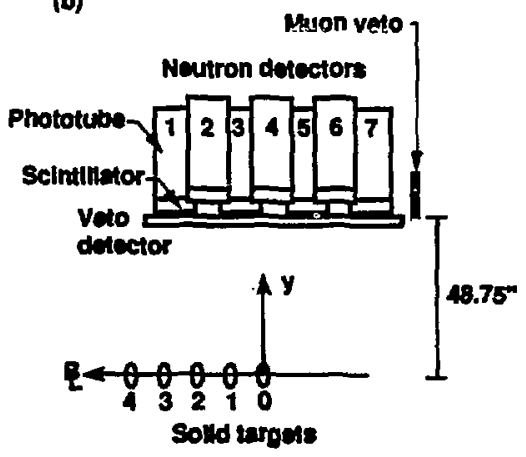

Figure 15. Arrangement of liquid scintillator neutron TOF detectors in FNAL E-665.

at FNAL con*isting of an array of 7 liquid scintillator neutron TOF detectors capable of $\gamma$-ray rejection through pulse-shape discrimination. (Figure 15). (The targets used by E665 in 1990 were $\mathrm{H}_{2}, \mathrm{D}_{2}, \mathrm{C}, \mathrm{Ca}, \mathrm{Pb}$.) The neutron detectors, which cover a range of angles from $80^{\circ}-140^{\circ}$ and sit $1.5-2.4 \mathrm{~m}$ from the solid target disks, are optimally suited for detecting neutrons at energies below $50 \mathrm{MeV}$, i.e. the statistical plus pre-equilibrium components of the decay.

The collaboration reports that true neutron signals have been seen in coincidence with deep-inelastically scattered muons, and they estimate a total coincident sample size of approximately $10^{3}$ per target for the 1990 run. Initial analysis of the neutron energy spectrum from $\mathrm{Pb}$ indicates that the nucleus is not left completely cold by the deepinelastic scattering. The data sample is sufficient to allow rough cuts in $Q^{2}, \nu$, ard $z$ of the detected forward-going hadrons. A quantitative analysis of these data is in progress [25].

\section{DONCLUSIONS}

If the study of hadronization is perceived to be a central component of the program, then it is important to design the accelerator with a suitably high energy, perhaps higher than was initially countenanced for this project. A reasonable minimum would seem to be $25 \mathrm{GeV}$. The point is to be sure to be able to connect to the regime where the formation length $\mathrm{er}$ for fast hadrons is aignificantly larger than that of a medium weight nucleus. Three points are important for conclusive measurements: (i) the systematic errors between nuclear targets must be kept very low (1\% if possible); (ii) the integrated luminosity should be high, of the order of $10^{40}$, in order to ensure sufficient statistics to do the separation described in small elements of $\Delta \nu \Delta z \Delta p_{\perp}^{2}$; and (iii) measurements of hadrons even down to $x_{F}=0$. In regard to this latter point, Gyulassy and Plümer [5] 
note that the MIT-SLAC data at low $x$ present a great puzzle in showing no evidence for any dramatic accretion due to cascading down; even the 'string-flip' model fails to adequately account for the data.

Direct photon measurements may be very promising, both inclusive and exclusive; this calls for a near- $4 \pi$ detector with good photon position and energy resolution. As always, hermeticity of the detector with as low a photon energy threshold as possible is important in minimizing backgrounds due to unreconstructed $\pi^{0}$. The availability of $e^{+}$ beams would be a great boon insofar as the $\sigma\left(e^{+}\right)-\sigma\left(e^{-}\right)$asymmetries most directly reveal the Compton physics at the amplitude and phase level, and these asymmetries are expected to be large. It would seem however, that only the storage ring option could avail of polarized positrons to complement polarized electrons, which is what one might ultimately want to fully exploit this program.

Finally, while conventional nuclear decay measurements have made little inroads so far in high energy electron scattering for the study of the target spectator, there may be much to be learned here. These techniques have been developed to a high degree in hadron scattering and heavy ion physics and could be fruitfully introduced to this discipline.

I wish to thank all my collaborators in the PEGASYS project, as the preparation of that proposal has done much to focus our thinking ebout the possibilities of a facility such as has been discussed at this workshop.

This work was performea under the auspices of the US Department of Energy by the Lawrence Livermore National Laboratory under contract no. W-7405-ENG-48.

\section{REFERENCES}

1 L. S. Osborne et al, Phys. Rev. Lett. 40 (1978) 1624.

2 W.J. Womersley, "A Study of Forward Hadron Production in Deep Inelastic MuonNucleus Scattering", Thesis, Oxford (1986); P. Renton, Oxford University Print $55 / 88$ (1988).

3 A. Bialas and M. Gyulassy, Nucl. Phys. B291 (1987) 793.

4 N. Pavel, proceedings of this workshop.

5 M. Gyulassy and M. Plümer, Nucl. Phys. B (to be published).

6 B. Kopeliovich, Phys. Lett B243 (1990) 141.

7 S. J. Brodsky, SLAC-PUB-4551 (March, 1988).

8 A.S. Carroll et al, Phys. Rev. Lett. 61 (1988) 1698.

9 R. Milner et al, SLAC proposal NE-18 (1990).

10 Bernard Pire and John P. Ralaton, in Proceedings of the Topical Conference on Electronuclear Physics with Internal Targets, 9-12 January, 1989, R.G. Arnold (ed.), World Scientific, 229.

11 S.J. Brodsky, SLAC-PUB-4387 (August, 1987). 
12 G.R. Farrar, H. Liu, L.L. Frankfurt, M.I. Strikman, Phys. Rev. Lett. 61 (1988) 686.

13 J.V. Geaga et al, Phys. Rev. Lett. 45 (1980) 1993.

14 L.L. Frankfurt and M.I. Strikman, Phys. Rep. 76 (1981) 215, and references therein.

15 J.P. Berge et al, Phys. Rev. D18 (1978) 1367.

16 E. Matsinos et al, Z. Phys. C44 (1989) 79.

17 M.I. Strikman, proceedings of this workshop.

18 V.I. Chernyak, A.A. Ogloblin, I.R. Zhitnitsky, Novosibirsk preprints INP. 87135,136 , and references therein.

19 S.J. Brodsky, J.F. Gunion, R.L. Jaffe, Phys. Rev. D6 (1972) 2487.

20 E. Maina and G. Farrar, Phys. Lett. B206 (1988) 120.

21 P. Kroll, M. Schürmann, W. Schweiger, WU-B-90-15 (1990).

22 G.R. Farrar and H. Zhang, Phys. Rev. Lett. 65 (1990) 1721; G.R. Farrar and H. Zhang, Phys. Rev. D41 (1990) 3348, and erratum, ibid. D42 (1990) 2413.

23 C. Hyde-Wright, private communication (1991)

24 J. Zingman and A.K. Kerman, private communication (1989).

25 K.A. Grifioen, F.S. Dietrich, H.C. Clark, K. Hicks, and R. Finlay, "Nuclear Decay Following Deep-Inelastic Scattering", E665 internal progress report, 13 Sept. 1990; K.A. Griffioen, "Nuclear Decay following Deep-Inelastic Muon Scattering," Talk at PEGASYS Workshop, Jan.9-12, 1991. 